\title{
The theory of relativity of motion: A new approach
}

\author{
Tahar Latrache \\ B. P. 129 Salem Lalmi, 40003 Khenchela, Algeria. \\ Accepted 28 March, 2013
}

\begin{abstract}
For more than a century, people's belief about special relativity and its formulas was, in fact, inaccurate, because Einstein fails by delaying in the wrong conception. However, one of these formulas is correct, by chance, but the others are incorrect. In the present approach, new expressions for time dilatation, composition of velocities, different masses (energy and inertial masses) and energy are demonstrated and presented theoretically and graphically in an exact and accurate manner, using exact equations of motion and good conception.
\end{abstract}

Key words: Relativity theory, relativity of motion, special relativity.

\section{INTRODUCTION}

The publication in 1687 of Newton's Principia laid down so satisfactory a foundation for further dynamical considerations, that it seemed as though the ideas of Galileo and Newton on the nature of space and time, employed there, would certainly remain forever suitable for the interpretation of natural phenomena. And indeed upon this basis has been built the whole structure of classical mechanics which, until our recent familiarity with very high velocities, has been found completely satisfactory for an extremely large number of very diverse dynamical considerations.

Since the year 1905 (Dragon, 2012), which marked the publication of Einstein's momentous article on the theory of relativity, the development of scientific thought has led to a complete revolution in accepted ideas as to the nature of space and time, and this revolution has in turn profoundly modified those dependent sciences, in particular mechanics and electromagnetic, which make use of these two fundamental concepts in their considerations (Dragon, 2012; Dunsby, 2000; Einstein, 1905; Einstein, 1920; Norton, 2004; Tolman, 2010).

In the following pages it will be our endeavor to present a description of our new notions as to the nature of space and time, and to give a partial account of the consequent modifications to Einstein's thought which have been introduced into various fields of science. Before proceeding to this task, however, we may well review the ideas and the postulates that are based on our perception of the relativity of moving bodies.

\section{Postulates of the relativity theory}

The first postulate of relativity as originally stated by Newton was that it is impossible to measure or detect absolute translator motion through space. No objections have ever been made to this statement of the postulate in its original form. In the development of this theory of relativity, the postulate has been modified to include the impossibility of detecting translatory motion through any medium or ether which might be assumed to pervade space.

The second postulate of relativity states that the velocity of light in free space appears the same to all observers regardless of the relative motion of the source of light and the observer. This postulate may be obtained by combining the first postulate of relativity with a principle which has long been familiar to the ether theory 


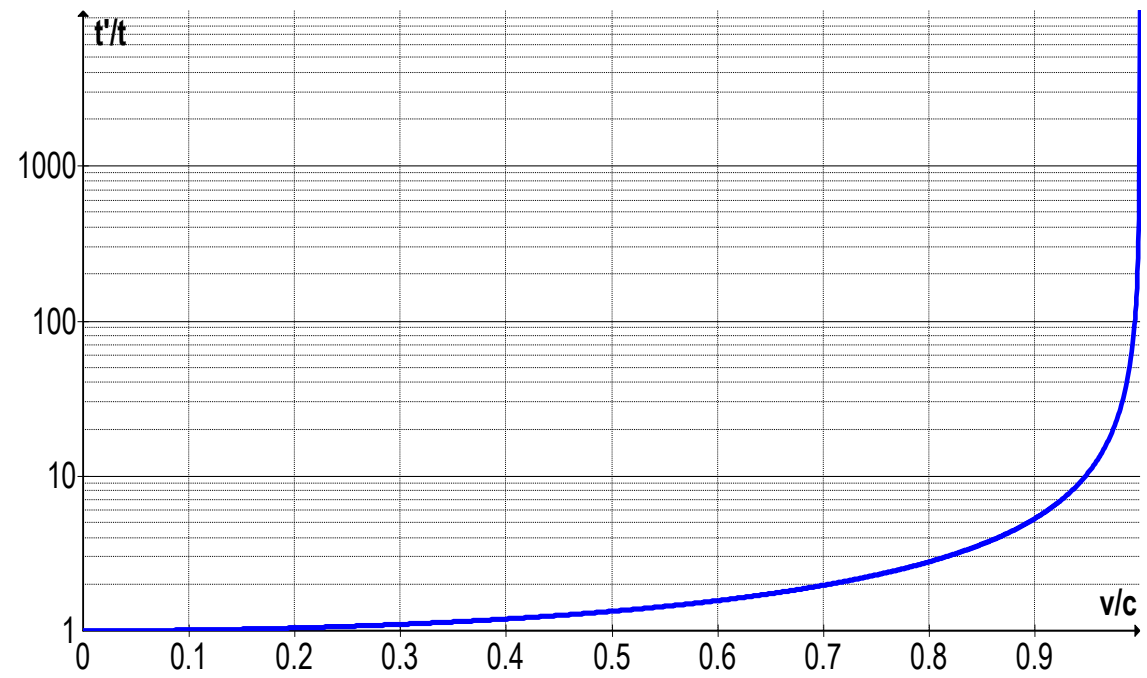

Figure 1. Variations of $\left(t^{t} / t\right)$ in function of $(v / c)$.

of light. This principle states that the velocity of light is unaffected by a motion of the emitting source; in other words, that the velocity with which light travels past an Observer is not increased by a motion of the source of light towards the observer. The first postulate of relativity adds the idea that a motion of the source of light towards the observer is identical with a motion of the observer towards the source. The second postulate of relativity is seen to be merely a combination of these two principles, since it states that the velocity of light in free space appears the same to all observers regardless of both the motion of the source of light and of the observer.

\section{THE TIME DILATATION}

First, we have to note that the stationary observer or system is nobody or nothing else than somebody or system at rest in comparison with somebody or system moving. Suppose that a bus moves with the velocity $v$, and an observer sites in the rear place of the bus and transmits a signal of light to a mirror placed towards the bus and returns back to the observer. The signal takes $t$ forward and $t$ back. The length of the bus is $I$. then, we can write:

$$
\mathrm{I}=\mathrm{ct}
$$

The length of the bus as seen by the stationary observer is I'. As seen by the stationary observer, the time used in taking the signal of light forward is $t 1$ and back is $t 2$ and the half of the tow times used in taking the signal forward and back as seen by the stationary observer is $t^{\prime}$. Then we can write the equation of motion of the bus as follows:

$\frac{1}{2}\left(\frac{T+w t_{2}}{\sigma}+\frac{\mathbb{T}-\omega t_{2}}{\sigma}\right)=t$
But

$I^{\prime \prime}=(c-w) t_{2}=(c+w) t_{2}$

Substituting l' by its expressions (3) in Equation (2), we can find:

$\frac{2}{2}\left(t_{1}+t_{2}\right)=t$

We know that, as seen by the stationary system,

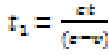

$t_{2}=\frac{m}{\| s+x]^{\mid}}$

Substituting $t 1$ and $t 2$ by their expressions (5) and (6) respectively in (4), we obtain:

$\frac{x^{x}}{\left[\xi^{x}-w^{1}\right]}=t$

or

$t^{\prime}=\frac{t}{\left.(1-(x / t)]^{2}\right)}$

Figure 1 shows the variation of $t / t$ according to the variations of $(\mathrm{w} / \mathrm{c})$.

\section{Length}

Substituting $t 1$ and $t 2$ by their Expressions (5) and (6) respectively in Equation (2), then:

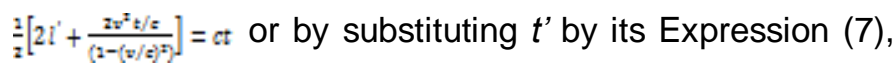
we obtain: 


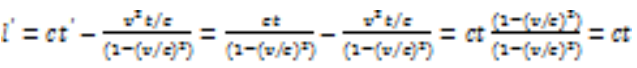

Substituting $c t$ by (Equation 1), we obtain:

$l^{\prime}=\mathbb{I}$

There is no changing then in the length according to the stationary system, and this is against Einstein's relativity.

\section{Composition of velocities}

Suppose a ball is shot by the observer who sites in the rear place of the bus (moving with the velocity $v$ ) by a velocity $\mathrm{w}$ relative to the bus, and bends on its way forward and back towards the bus. The ball then moves in accordance with the equations:

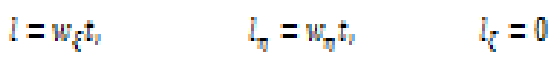

The ball takes $t$ forward and back from the front of the bus as seen by the moving observer. But, the ball takes the rates $\frac{w}{[x-x]]}$ forward and $\frac{w}{[x+x]}$ back as seen by the stationary observer. The difference of time between the clocks of the stationary and the moving observers as seen by them is:

$\frac{1}{2}\left[\frac{[+V t}{(s-w)}-\frac{T+V t}{(s+w)}\right]$

Then the time $t^{\prime}$ as seen by the stationary observer can be written as:

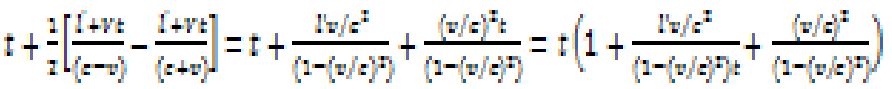

Substituting $=\mathbb{I}$, then the equation becomes:

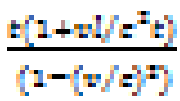

Substituting Equation (9) in this last equation, then we can obtain the time interval for the ball moving from one end of the bus forward and back with the velocity $\mathrm{w}_{\varepsilon}$ as seen by the stationary observer:

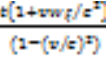

Hence, the stationary observer finds that the ball is moving past the bus by the velocity

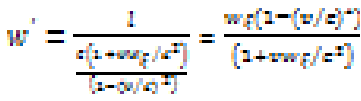

This makes the total velocity used by the ball to past itself equal to the sum:

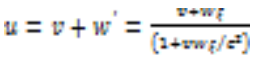

Then,

$x^{x}=\frac{w+w_{E}}{2+w w g^{3} x^{x}} t^{\prime}$

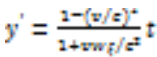

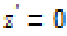

If we place,

$\mathbb{x}^{2}=u_{x}^{2}+\mathbb{u}^{2}+\mathbb{u}^{2}=\left(\frac{d x^{2}}{d t^{2}}\right)^{2}+\left(\frac{d y^{2}}{d t^{2}}\right)^{2}+\left(\frac{d v^{2}}{d t^{2}}\right)^{2}$

$W^{2}=w_{\frac{2}{8}}^{2}+w_{0}^{2}+w_{q}^{2}$

$\alpha=\tan ^{-1}\left(w_{q} / w_{q}\right)$

$\approx$ is then to be looked upon as the angle between the velocities $\mathrm{E}$ and $\mathrm{w}$. After a simple calculation, we obtain:

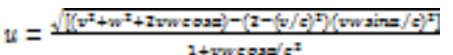

The variations of $\mathrm{w}$ when $\mathrm{v}$ and $\mathrm{w}$ varied, for different values of $\alpha$ are shown in Figure 2. If $\mathrm{w}$ also has the direction $x$ of the bus, we get

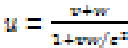

The variation of $\mathrm{a}$ according to the variation of $\mathrm{v}$ and $\mathrm{w}$ is represented in Figure 3.

\section{MASSES AND FORCE}

We predict that the mass of a fixed quantity of matter, spatially in motion with a velocity $V_{e}$ is greater than when it is at rest. For this to be so, the increase in mass can only take place during the time spatial acceleration is in effect. This process can be investigated by treating mass as a variable when analyzing the change in the existence of momentum of a point mass subjected to a spatial acceleration in the stationary system $\mathrm{K}$. If $\mathrm{m}$ is the mass of the point mass with existent velocity $\mathrm{V}$ in $\mathrm{K}$, then its existent momentum will be given by:

$M=m V=\left[V+j c\left(\frac{d r}{d t}\right)\right]$

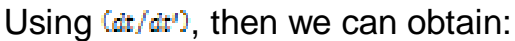

$M=m V=\left[W+j \in\left[1-[w / c]^{*}\right\rfloor\right\rfloor$ 
(a)

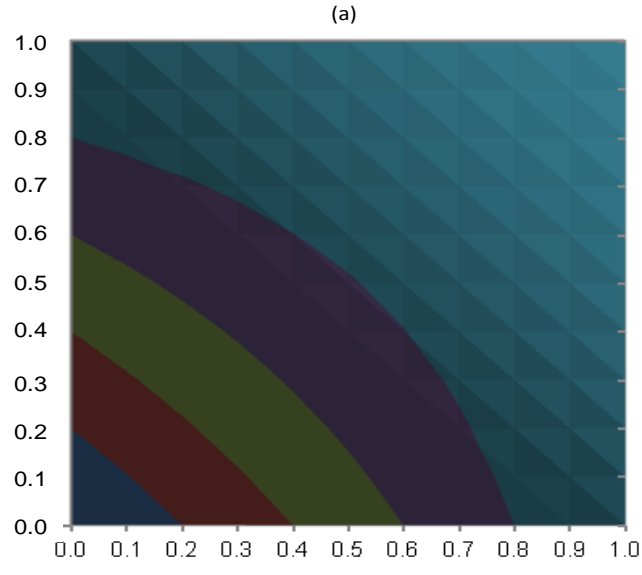

(c)

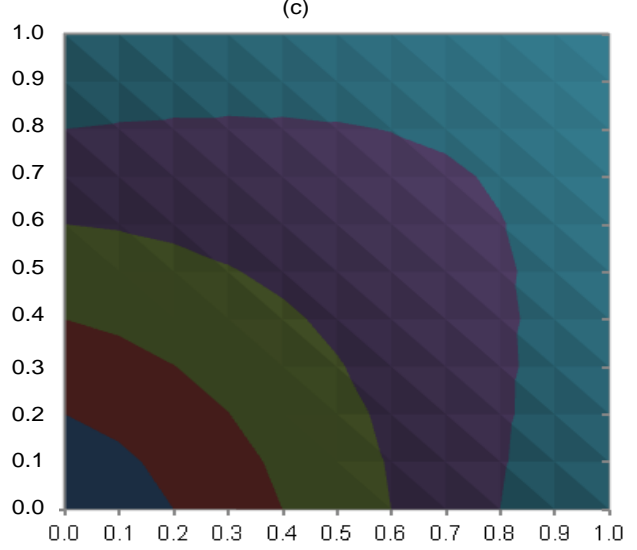

(b)

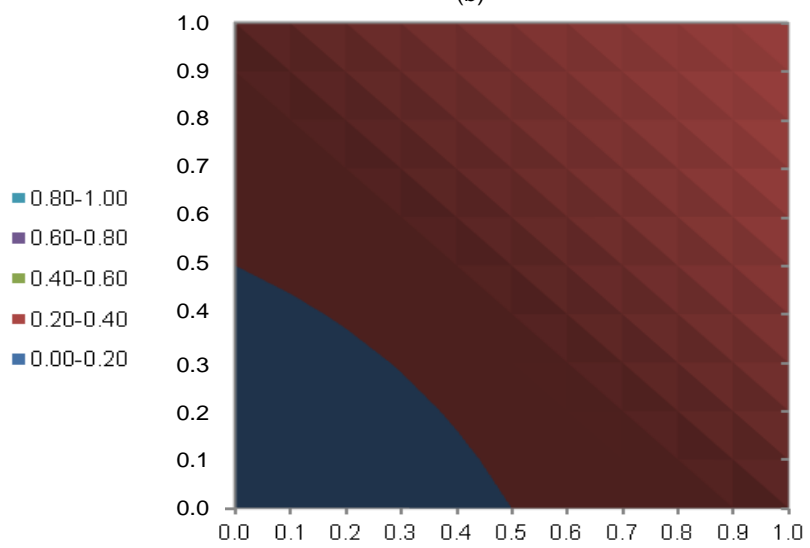

$=0.50-1.00$

$=0.00-0.50$

Figure 2. Variations of $(\mathrm{w} / \mathrm{c})$ in function of $(w / \alpha)$ and $(w / c)$ for different values of $\alpha$ : a) $\alpha=\pi / 8$, b) $\alpha=\pi / 4$, c) $\alpha=3 \pi / 8$, d) $\alpha=\pi / 2$.

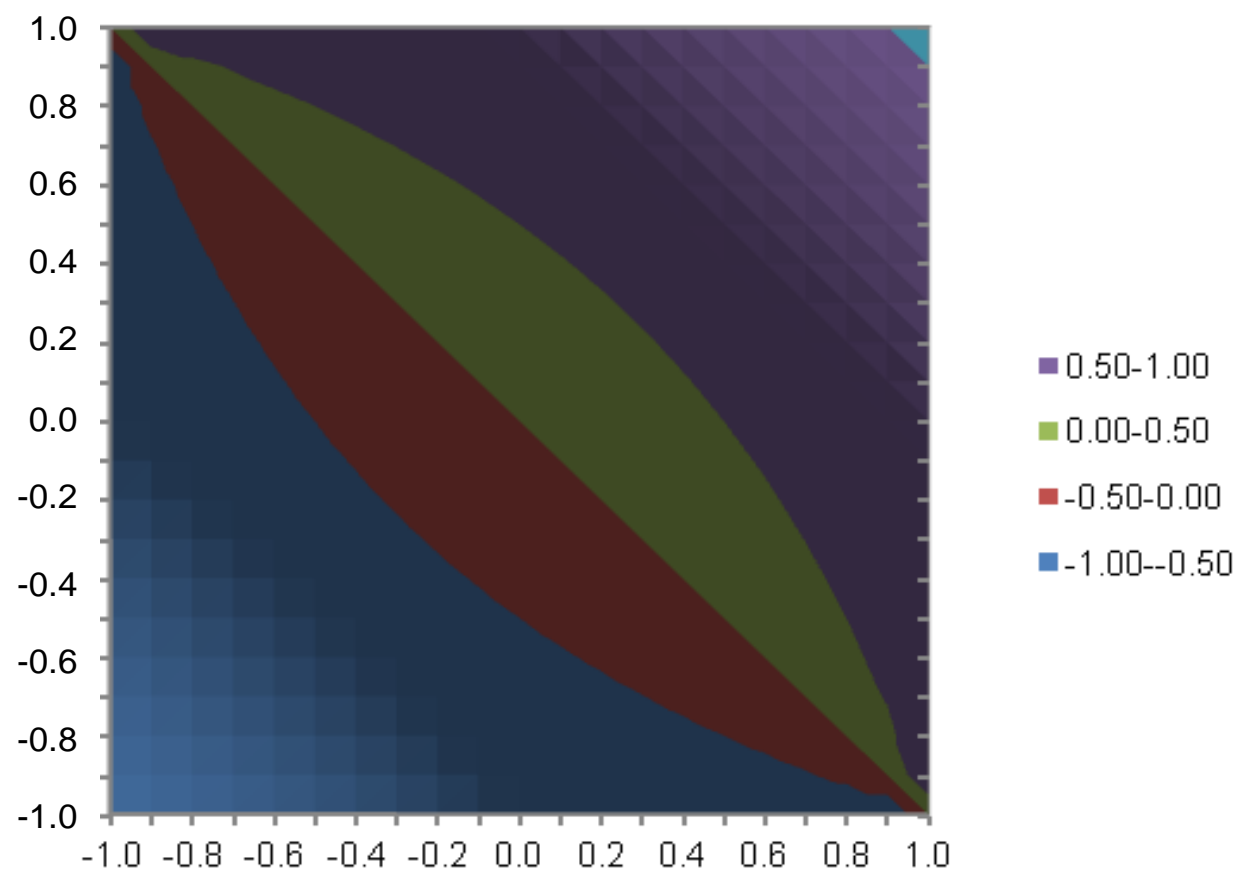

Figure 3. Variations of $(u / c)$ in function of $(v / c)$ and $(w / c)$. 
If $F$ is the force applied to effect acceleration, then:

$F=\frac{a v}{a}=m b+m_{n}+j \sigma\left(m\left(1-(\omega / c)^{2}\right)-2 m v b / c^{2}\right)$

It shows that there are four kinetic reactions involved in this process.

If $F$ is purely spatial, the temporal component will be zero, whereby:

$\operatorname{mot}\left(1-(\omega / c)^{2}\right)=2 \operatorname{mov} / c^{2}$

So that upon separating variables and integrating,

$\operatorname{In}(m)=-\operatorname{In}\left(1-(w / c)^{2}\right)+\mathbb{k}$

The constant of integration is obtained from initial conditions: when $v=0, m=m_{g}$ the mass of the point mass when spatially at rest in $\mathrm{K}$. That is, the 'rest mass'. Then:

$k=\operatorname{In}\left(m_{\theta}\right)$

This gives in Equation (20):

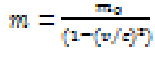

$m$ represents the energy mass.

Substituting Equation (21) for Equation (19), yields:

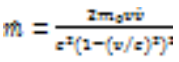

This thus represents the time rate of change of mass subjected to spatial acceleration in $\mathrm{K}$. These last two terms, Equations (21) and (22), may now be inserted into Equation (18), whereupon the temporal component vanishes, and, if rectilinear motion only is being considered, $F$ can also be reduced to special vector, so that Equation (18) is obtained, after reduction:

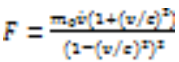

As $F$ is arbitrary, Equation (23) represents the spatial rectilinear equation of motion of a point mass in $\mathrm{K}$. Note that the right hand side of Equation (23) is the product of the spatial acceleration and a mass term. Putting:

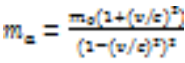

Then $m_{m}$ is, from Equation (23), synonymous with inertial mass. The variations of $m$ and $m_{m}$ according to the variation of $w / c$ are represented in Figure 4.

\section{Energy}

From the results of the preceding section, energy can now be demonstrated as follows.

Let us consider the change in energy of the point mass as the spatial acceleration proceeds. Integrating Equation (23) in respect to the spatial distance travelled, we get,

$E=\int F d r$

$E=\int m=\frac{w}{d t} d r$

This from Equation (24) becomes:

$E=m_{0} c^{2} \int \frac{\left[1+(w / c)^{2}\right)(w / c]}{\left(1-(w / c)^{2}\right)^{2}} d\left(\frac{E v}{\sigma}\right)$

Using the mathematics, this is evaluated as:

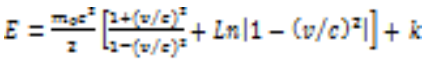

Initial conditions are that $E=0$ when $v=0$ so that $k=-m_{\mathrm{q}} \mathrm{E}^{2} / 2$. Inserting this into Equation (25) then $E$ becomes:

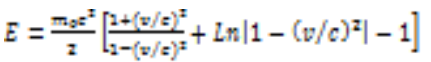

Or, after simplification:

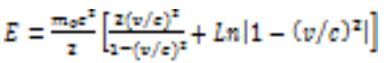

We can observe in Figure 5 variation of $E$ according to the variation of $[w / c]^{2}$. At the neighborhood of $[w / c)=0$ :

$\operatorname{Ln}\left|1-(w / c)^{2}\right|=-(w / c)^{2}+\cdots$

Substituting this expression in Equation (26), then for moderate values of the velocity we can obtain and use the expression:

$E=\frac{1}{2} m_{0} w^{2}$

which represents the expression of the classical kinetic energy and presents an approximation of the relation given by Equation (26) for moderate velocities.

\section{COMPARISON WITH EXPERIMENTAL RESULTS}

The satellite orbiting at the distance $36,623,583.565 \mathrm{~m}$ from the surface of the earth and at $43,001,583.565 \mathrm{~m}$ from its center has the rate of time 0.000048 s/day reported to earth day which is $86,164 \mathrm{~s}$; then its orbiting time for one earth day is $86,164.000048 \mathrm{~s}$. The velocities 


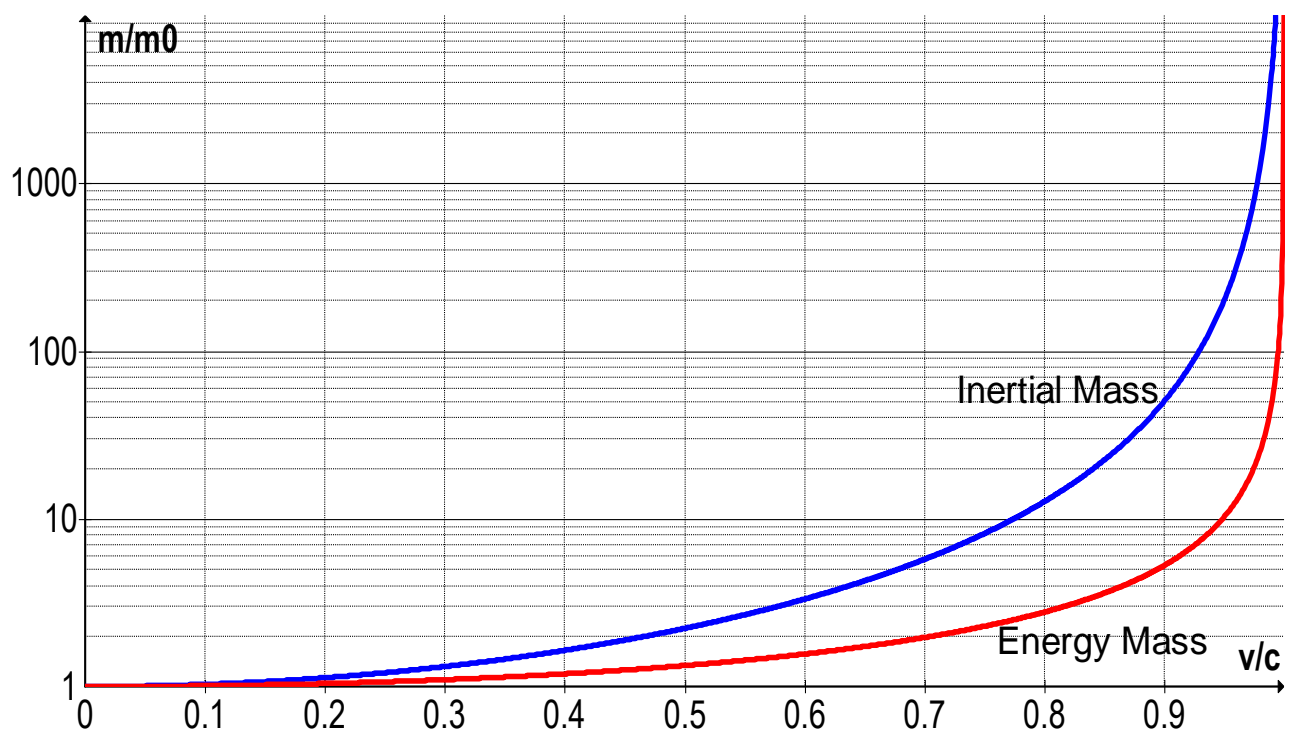

Figure 4. Variations of $\left(m / m_{0}\right)$ and $\left(m_{m} / m_{0}\right)$ in function of $(v / c)$.

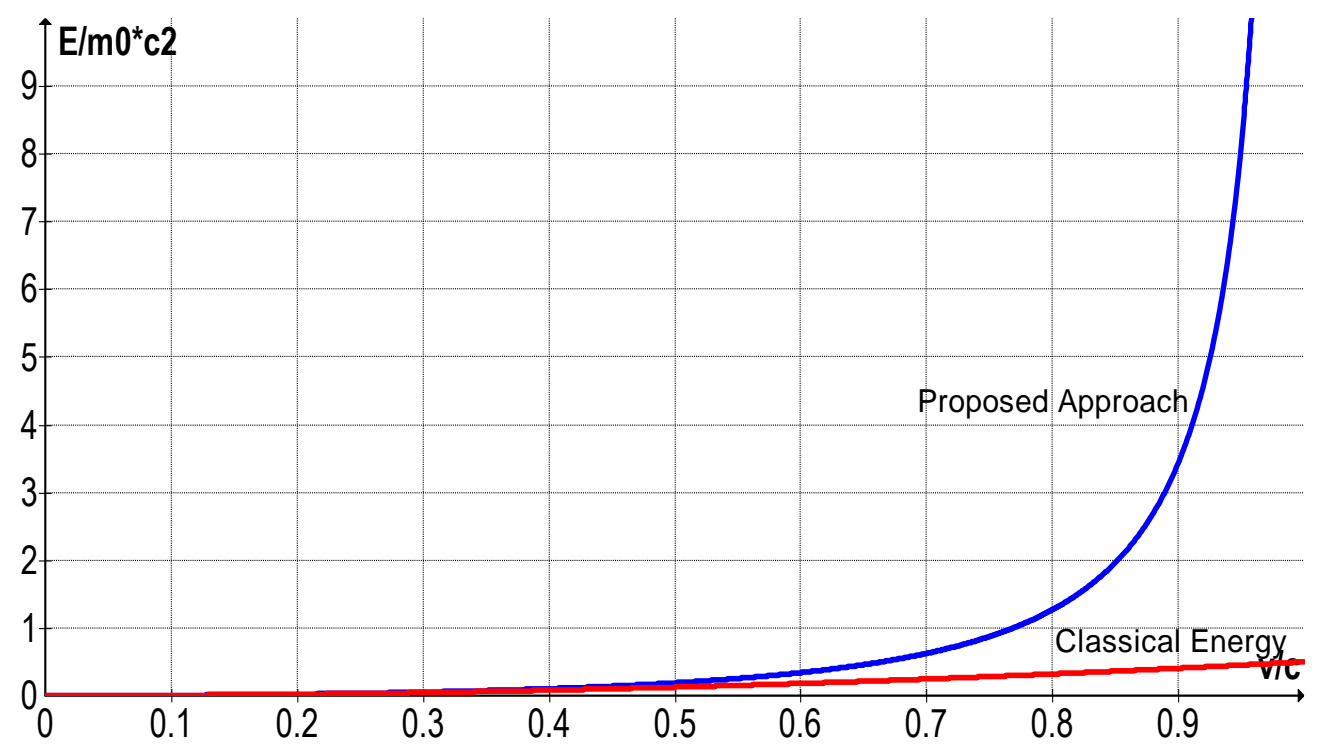

Figure 5. Variations of the proposed energy and the classical energy $\left(\frac{1}{2} m_{0} v^{2}\right)$ in function of $[v / c)$.

of the earth and of the satellite reported to the center of the earth are 465.092 and $3,135.729 \mathrm{~m} / \mathrm{s}$, respectively. Using Equation (16), we can obtain the velocity of the satellite reported to the surface of the earth, $\mathrm{w}=(u-w)\left(1-\left(w / c^{2}\right)\right)$ and then numerically equals to $2670.637 \mathrm{~m} / \mathrm{s}$. The difference between satellite time and earth time as seen by a stationary person at earth is then equals to $-(\mathrm{w} / \mathrm{c} / \mathrm{C})^{\pi}$ per unit of earth time. Using Equation (7), numerically equals to per earth day, multiply the last expression with $86,164,000,000$ micro-seconds to find 6.83 micro-seconds which represent the difference between satellite time and that of earth day as seen by a stationary observer on earth and according to the relativity of motion presented and -7 micro-seconds measures. We can remark that the difference between the theory and the experiment does not exceed $20 \%$ micro-seconds per earth day.

\section{Conclusion}

The proposed theory is accurate based on conception 
and formulation. In this approach, new expressions for time dilatation, addition of velocities, energy and inertial masses and energy are presented theoretically and graphically. We can remark that for ordinary velocities, the expressions of these different quantities obtained are approximately applicable with the classical mechanics expressions which ameliorate the exactitude and the generalization of our approach. The comparison between earth and satellite times represented by the difference of time between the two times present a good agreement between the theoretical and experimental results, which demonstrate that the approach proposed is mathematically and physically good.

\section{REFERENCES}

Dragon N (2012). Geometry of special relativity. Internet Document. Website: www.itp.uni-hannover.de/ dragon/rel_e.pdf.

Dunsby PKS (2000). An introduction to tensors and relativity. Internet Document. Website: www.mth.uct.ac.za/omei/gr/notes.ps.gz.

Einstein A (1905). On the electrodynamics of moving bodies. Ann. Phys. 17:891.

Einstein A (1920). Relativity: The Special and General Theory. Methuen and Co Ltd.

Norton JD (2004). Einstein's theory of relativity and the problems in the electrodynamics of moving bodies that led him to it. Cambridge University Press.

Tolman RC (2010). The theory of the relativity of motion. EBook. 